\title{
Chatbots na educação: uma Revisão Sistemática da Literatura
}

Neiva Larisane Kuyven - PPGIE/UFRGS , UNIFTEC, neivakuyven@acad.ftec.com.br Carlos André Antunes - UNIFTEC, c4rlo4@gmail.com Vinicius João de Barros Vanzin - UNIFTEC, vini_vanzin@ hotmail.com João Luis Tavares da Silva - UNIFTEC, joaoluis.tavares@ gmail.com Aliane Loureiro Krassmann - PPGIE/UFRGS, alkrassmann@gmail.com Liane Margarida Rockenbach Tarouco - PPGIE/UFRGS, liane@ penta.ufrgs.br

Resumo: Um chatbot é um sistema capaz de conversar com um usuário em linguagem natural, de maneira a simular um diálogo humano. Exemplos de chatbots podem ser encontrados nas áreas de saúde, entretenimento, negócios e educação. Este artigo apresenta uma Revisão Sistemática da Literatura acerca da aplicação dos chatbots para fins educacionais em bases de dados nacionais e internacionais. Por meio da análise, constatou-se que a maior parcela dos chatbots foca na área de conhecimento da Ciência da Computação e que é destinada para o nível de ensino superior. Em geral, os estudos reportaram resultados positivos na construção do conhecimento dos alunos e destacaram os principais fatores que poderiam ser aprimorados para maior consistência do diálogo.

Palavras-chave: chatbots, agentes conversacionais, inteligência artificial, educação.

\section{Chatbots in education: a Systematic Literature Review}

\begin{abstract}
A chatbot is a system capable of conversing with a user in natural language, in order to simulate human dialogue. Examples of chatbots can be found in the areas of healthcare, entertainment, business and education. This paper presents a Systematic Literature Review on the application of chatbots for educational purposes in national and international databases. Through the analysis, it was found that the largest share of chatbots focuses on the area of knowledge of Computer Science and is intended for higher education level. In general, studies have reported positive results in the construction of students' knowledge and highlighted the main factors that could be enhanced for greater dialogue consistency.
\end{abstract}

Keywords: chatbots, conversational agents, artificial intelligence, education.

\section{Introdução}

Um chatbot, também encontrado na literatura como chatterbot ou agente conversacional, é um software capaz de comunicar-se com uma pessoa em linguagem natural (Moraes; De Souza, 2015). A criação dos chatbots remonta ao sistema de conversação ELIZA, que emulava um psicoterapeuta (Weizenbaum, 1966). Com o passar dos anos, novas técnicas de Inteligência Artificial (IA) foram aplicadas à construção desses agentes, de modo que os exemplos de sistemas podem ser categorizados, de forma geral, em três paradigmas ou "gerações": a primeira, baseada no casamento de padrões e regras gramaticais; a segunda, fundamentada nas regras de produção e nas redes neurais artificiais; e a terceira, que faz uso das linguagens de marcação AIML ${ }^{1}$. (Sgobbi et al., 2014).

Aplicações de chatbots variam desde e-commerce, recuperação de informações, helpdesk, suporte ao cliente e assistentes digitais, entre outros (De Gasperis et al., 2013). Masche e Le (2017) sugerem que a interação entre humanos e sistemas de computador está mudando para interfaces baseadas em linguagem natural. Devido ao objetivo

${ }^{1}$ Artificial Intelligence Markup Language. Projeto mais conhecido é o A.L.I.C.E 
fundamental da Inteligência Artificial(IA) de criação de máquinas com capacidade de comunicação com humanos (Leonhardt et al., 2003) e à constatação da preferência dos usuários em utilizar sistemas em linguagem natural, verifica-se a popularização dos chatbots e ferramentas associadas (Moraes; De Souza, 2015). Por isso, é crescente a quantidade de artigos sobre o tema, inclusive no Brasil, abrindo espaço para Revisões Sistemáticas de Literatura (RSL) com vistas a avaliar o estado da arte sob diferentes enfoques.

Soliman e Guetl (2010) propõem uma RSL sobre agentes pedagógicos inteligentes inseridos em ambientes virtuais de aprendizado. Sewald Junior et al. (2011) analisam os principais problemas de fluidez de conversação nos chatbots, restringindo-se a agentes em língua portuguesa. Bradeško e Mladenić (2012) comparam e discutem as tecnologias empregadas pelos chatbots vencedores do Prêmio Loebner, observando a evolução ao longo dos anos. Dale (2017) investiga o cenário comercial da área de processamento de linguagem natural, da qual os sistemas conversacionais fazem parte. Bernardini, Sônego e Pozzebon (2018) propõem uma análise das pesquisas a nível mundial, inerente à temática chatbots, através de uma análise bibliométrica qualitativo-quantitativa.

No entanto, observa-se que as RSL existentes focam em contextos diferentes deste trabalho, que tem por objetivo um estudo sobre a aplicação dos chatbots para fins educacionais. Para compreender a aplicação, utilização e resultados de chatbots como suporte educacional, esta pesquisa apresenta uma RSL. O artigo está estruturado da seguinte forma: a Seção 2 apresenta o método de pesquisa, na Seção 3 são descritos os resultados obtidos e as discussões, e por fim, a Seção 4 apresenta as conclusões e trabalhos futuros.

\section{Método de pesquisa}

A Revisão Sistemática de Literatura segue a proposta de Kitchenham et al. (2007), que definiu uma metodologia para avaliar e interpretar pesquisas primárias relevantes. As etapas desta RSL resume-se em três: planejamento, condução e análise dos resultados, e são a seguir apresentadas.

1. Planejamento: Com o objetivo de fornecer uma visão geral das pesquisas que vêm sendo conduzidas a respeito de chatbots aplicados à educação, foram formuladas as seguintes Questões de Pesquisa (QP): QP1: Quais são as bases de dados com o maior número de artigos publicados? QP2: Há instituições que se destacam em pesquisas sobre chatbots em educação? QP3: Em quais níveis educacionais são aplicados os chatbots? QP4: Quais são os temas mais pesquisados no contexto dos chatbots na educação? QP5: Quais são os objetivos do uso de chatbots na educação? QP6: Quais são as principais técnicas e tecnologias que vêm sendo utilizadas na implementação de chatbots na educação? QP7: Quais são os principais resultados observados nos trabalhos analisados?

Foram definidos os idiomas de busca português e inglês, as questões de pesquisa, as bases de dados a serem consultadas, a estratégia de busca e os critérios de inclusão e exclusão. O período de abrangência foi definido entre os anos de 2000 e 2017, cobrindo 18 anos de pesquisas.

2. Condução: a seleção dos artigos para buscar respostas às QP foi conduzida de acordo com os passos a seguir descritos.

a) Identificação de bases de dados mais relevantes relacionados ao tema: os veículos de publicação científica (anais de eventos e periódicos) que foram pesquisados constam no Quadro 1. A seleção engloba alguns dos principais canais nacionais e internacionais relacionados à área de informática na educação, caracterizados, em sua maioria, por classificação no sistema de avaliação interdisciplinar no Qualis CAPES. Das 12 bases de 
dados selecionadas, cinco são de abrangência nacional e sete, de abrangência internacional.

Quadro 1 - Bases de dados selecionadas para a RSL

\begin{tabular}{|l|c|}
\hline \multicolumn{1}{|c|}{ Bases de dados/Qualis } & Qualis CAPES \\
\hline ACM Transactions on Computer-Human Interaction (TOCHI) & A2 na Ciência da Computação \\
\hline Congresso Internacional de Informática Educativa (TISE) & B5 na Interdisciplinar \\
\hline Computers \& Education & A2 na Interdisciplinar \\
\hline Creative Education & B1 na Interdisciplinar \\
\hline IEEE Revista Iberoamericana de Tecnologias del Aprendizaje & B3 na Interdisciplinar \\
\hline IEEE Transactions on Learning Technologies & A2 na Educação \\
\hline Revista Latinoamericana de Tecnología Educativa (RELATEC) & B1 na Interdisciplinar \\
\hline \multicolumn{1}{|c|}{ Bases de dados nacionais } & B1 na Interdisciplinar \\
\hline Informática na educação: teoria \& prática & B1 na Interdisciplinar \\
\hline Revista Novas Tecnologias na Educação (RENOTE) & B1 na Ciência da Computação \\
\hline Simpósio Brasileiro de Informática na Educação (SBIE) & B3 na Ciência da Computação \\
\hline Workshop de Informática na Escola (WIE) & B3 na Ciência da Computação \\
\hline Workshop sobre Educação em Computação (WEI - CSBC) & \\
\hline
\end{tabular}

Fonte: os autores.

b) Busca de artigos nas bases de dados selecionadas: com relação a busca de artigos nas bases de dados apresentados no Quadro 1, para os canais que dispõem de mecanismo de busca em seus websites, foram pesquisadas palavras-chave combinadas com operadores lógicos, compondo a seguinte string de busca:

(chatbot OR chatterbot OR conversational agent $\mathrm{OR}$ agente conversacional $\mathrm{OR}$ agentes conversacionais) AND (education OR learning OR teaching OR educação OR ensino OR aprendizagem)

O operador "OR" é usado para indicar termos alternativos e o operador "AND" é usado para conectar termos.

Para os portais que somente disponibilizam os arquivos em formato PDF, foi realizada uma busca manual através da ferramenta de busca do Adobe Reader com as palavras-chave "chatbot", "chatterbot", "agente conversacional" e "conversational agent". Na ocorrência de uma dessas palavras-chave em um artigo (exceto na seção de referências), o mesmo já era selecionado. Com essa busca inicial, identificou-se um total de 159 artigos.

c) Aplicação dos critérios de exclusão e inclusão: na sequência, os critérios de exclusão e inclusão apresentados no Quadro 2 foram aplicados aos 159 artigos selecionados. A primeira análise foi conduzida pela leitura do título, resumo, palavras-chave, método, resultados e conclusões (ou equivalentes).

Quadro 2: Critérios de exclusão e inclusão da RSL

\begin{tabular}{|c|c|}
\hline Critérios de Exclusão (CE) & Critérios de Inclusão (CI) \\
\hline $\begin{array}{l}\text { CE1: O texto completo do artigo não está disponível. } \\
\text { CE2: Artigos em idioma diferente do inglês ou português. } \\
\text { CE3: Estudos secundários, relatórios técnicos, resumos ou } \\
\text { revisões sistemáticas. } \\
\text { CE4: Não aborda a aplicação propriamente dita de chatbots } \\
\text { na educação. } \\
\text { CE5: Não apresenta resultados claros e/ou os instrumentos } \\
\text { utilizados na avaliação dos chatbots. }\end{array}$ & $\begin{array}{l}\text { CI1: Artigos que apresentam chatbots } \\
\text { aplicados na educação, } \\
\text { independentemente da plataforma, área } \\
\text { e nível de estudo. } \\
\text { CI2: Artigos com resultados } \\
\begin{array}{l}\text { consistentes com os objetivos de } \\
\text { pesquisa. }\end{array}\end{array}$ \\
\hline
\end{tabular}

Fonte: os autores. 
3. Análise dos resultados: foi realizada a leitura e verificação dos artigos selecionados para garantir a pertinência dos materiais extraídos com a temática pesquisada. Após a aplicação dos critérios de inclusão e exclusão, a quantidade de artigos foi reduzida para 16, que representam, portanto, o conjunto final de trabalhos analisados de forma completa para responder às questões de pesquisa. Os artigos encontram-se distribuídos em maior número nos anos de 2012 a 2017, totalizando 11 artigos, representando 68,8\% das publicações. Destes, 2012 teve o maior número de publicações, totalizando em 3 artigos, representando $18,8 \%$ do total das publicações analisadas. Os artigos se dividiram igualmente entre língua inglesa e portuguesa, com 8 publicações em cada língua.

A análise das publicações extraídas, juntamente com as discussões, é exposta na seção a seguir. A listagem completa pode ser consultada em <https://goo.gl/VcmDJ9>.

\section{Resultados e discussões}

A leitura completa dos 16 artigos extraídos permitiu a coleta de informações relevantes às Questões de Pesquisa investigadas por meio da RSL. Além disso, técnicas de mineração de textos foram aplicadas no corpus resultante, contendo um total de 107.945 palavras.

Para realização da mineração de textos cada publicação extraída foi copiada para em um mesmo arquivo no formato .txt, onde retirou-se os nomes dos autores, agradecimentos e referências. Utilizou-se a ferramenta online gratuita Voyant Tools ${ }^{2}$, que permite desconsiderar da análise as stopwords (como adjetivos, artigos e advérbios, exemplo "a" "de" "esse") presentes no corpus. A ferramenta foi manualmente configurada com um rol de stopwords tanto em língua inglesa quanto portuguesa.

Identificou-se que os 10 termos mais recorrentes no corpus foram: learning (987), students (662), agente (278), training (272), test (264), support (259), styles (254), tutorial (224), agent (216), style (216). Observa-se que a maioria das palavras é da língua inglesa. Isso se deve ao fato de que o corpus, apesar de dividido igualmente quanto à quantidade de publicações ( 8 inglês -8 português), em termos de número de palavras é composto na sua maioria pela língua inglesa (77.580 palavras - 72\%). Já a língua portuguesa foi responsável por 30.365 palavras $(28 \%)$.

Para possibilitar uma análise mais acurada, o corpus foi dividido de acordo com a língua. $\mathrm{O}$ corpus em português teve as seguintes cinco palavras mais recorrentes: agente (278), conhecimento (165), aprendizagem (126), alunos (115), estudantes (98). Já na língua inglesa, obteve-se como cinco palavras mais recorrentes os termos: learning (967), students (659), training (272), test (263), support (255). A Figura 1 ilustra a nuvem de palavras referente ao corpus da língua portuguesa (à esquerda) e inglesa (à direita).
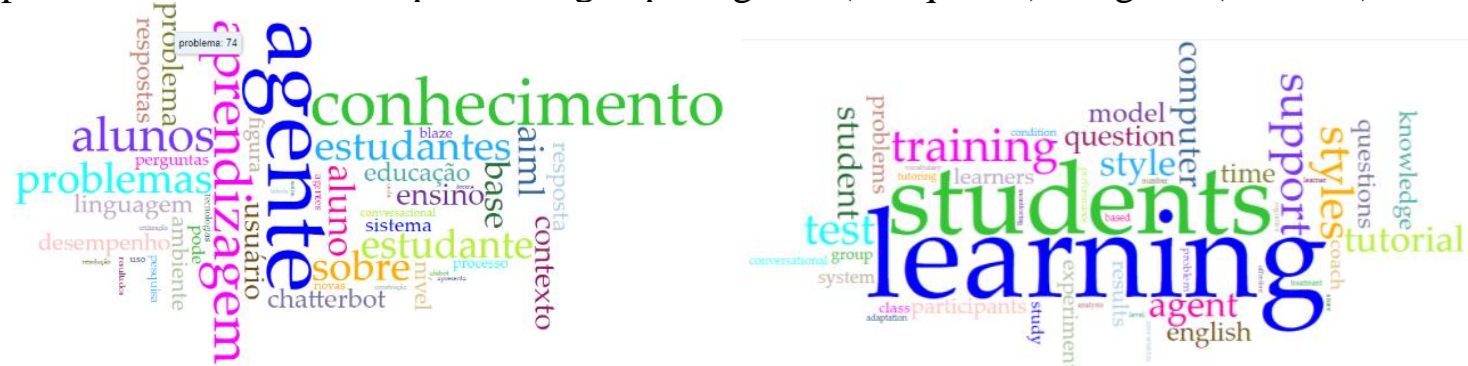

Figura 1: Nuvem de palavras com os artigos da RSL: em português (à esquerda) e em inglês (à direita).

A análise via mineração de textos permite ter uma visão geral do corpus que será analisado nesta RSL, seu conteúdo e contribuições, de forma automatizada, rápida e sucinta. A seguir, são apresentados e discutidos os resultados obtidos para cada QP.

\footnotetext{
${ }^{2}$ https://voyant-tools.org/
} 
3.1 QP1: Quais são as bases de dados com o maior número de artigos publicados? As seis bases de dados que deram origem às publicações extraídas na RSL, em ordem decrescente, foram: Computers \& Education (5-31,3\%); Revista Novas Tecnologias na Educação - RENOTE (4 - 25,0\%); IEEE Transactions on Learning Technologies (2 12,5\%); Simpósio Brasileiro de Informática na Educação - SBIE (2 - 12,5\%); Congresso Internacional de Informática Educativa - TISE (2 - 12,5\%); Revista Latinoamericana de Tecnología Educativa - RELATEC - $(1-6,2 \%)$.

Portanto, nesta RSL destacam-se a revista Computers \& Education, classificada no conceito A2 Interdisciplinar (Qualis CAPES), com aproximadamente 32\% dos artigos selecionados, seguida pela RENOTE - B1 Interdisciplinar, responsável por 25\% dos artigos selecionados. Assim, é possível observar também a saliência de duas bases de dados nacionais no contexto pesquisado, RENOTE e SBIE, responsáveis, juntas, por aproximadamente $37 \%$ das publicações extraídas.

3.2 QP2: Há instituições que se destacam em pesquisas sobre chatbots em educação? Dentre os 16 artigos analisados, observou-se a contribuição de autores de 23 instituições diferentes, sendo 16 delas nacionais e 7 internacionais. Nesse cenário, a instituição destaque é a Universidade Federal do Rio Grande do Sul (UFRGS), com a participação 6 publicações, seguida pela Manchester Metropolitan University (Reino Unido) e a Universidade de Cruz Alta (UNICRUZ) com participação em 2 artigos cada.

Sob outro enfoque, levando-se em consideração a origem do autor principal (primeiro autor) de cada publicação, as instituições de ensino que se destacaram foram: UFRGS, 2 - 12,5\%; Manchester Metropolitan University, 2 - 12,5\%; e UNICRUZ, 2 12,5\%). As demais instituições foram responsáveis uma única publicação (1- 6,2\%), sendo elas: Columbia University, Peking University, Aristotle University of Thessalonik, Vrije Universiteit Amsterdam, Carnegie Mellon University, Universidade Estadual de Londrina (UEL), Universidade Federal de Pernambuco (UFPE), Instituto Federal do Rio Grande do Norte (IFRN), Universidade de São Paulo (USP), Centro Universitário UNILASALE e Instituto Federal Fluminense (IFFluminense).

\subsection{QP3: A quais níveis educacionais são aplicados os chatbots?}

No que diz respeito ao nível educacional do público-alvo ao qual se destinam os chatbots dos estudos analisados, a maior parcela $(7-43,7 \%)$ corresponde aos agentes desenvolvidos para o ensino superior. Em segundo lugar (3 - 18,8\%), estão os chatbots aplicados no ensino médio. Nos níveis do ensino fundamental, pós-graduação e adaptável a vários níveis identificou-se 2 artigos (12,5\%) em cada.

Quadro 3 - Aplicação de chatbots por nível educacional

\begin{tabular}{|c|c|c|c|}
\hline Nível Educacional & ID & Quantidade de publicações & $\%$ \\
\hline Ensino Superior & $2,4,10,12,13,15$ e 16 & 7 & 43,7 \\
\hline Ensino Médio & 7,8 e 9 & 3 & 18,8 \\
\hline Ensino Fundamental & 1 e 3 & 2 & 12,5 \\
\hline Pós-graduação & 5 e 14 & 2 & 12,5 \\
\hline Adaptável a vários níveis (ensino geral) & $6 \mathrm{e} 11$ & 2 & 12,5 \\
\hline Total & & 16 & 100,0 \\
\hline
\end{tabular}

Fonte: os autores.

3.4 QP4: Quais são as áreas de conhecimento mais pesquisadas no contexto de chatbots na educação?

Analisando-se os artigos extraídos, identificou-se as áreas de conhecimento e as grandes áreas onde os estudos foram aplicados e/ou avaliados. Analisando as grandes áreas, 
verifica-se no Quadro 4, que a maioria dos estudos $(10-62,6 \%)$ encontram-se na Ciências Exatas e da Terra, seguido pela Linguística, Letras e Artes $(3-18,8 \%)$. Na sequência, Ciências da Saúde, Ciências Biológicas e Ciências Humanas com 6,2\% (1 publicação) cada.

Quadro 4 -Áreas de conhecimento abordadas nas publicações extraídas

\begin{tabular}{|c|c|c|c|c|}
\hline Área de Conhecimento & Grande área & $\begin{array}{c}\text { Quantidade de } \\
\text { publicações }\end{array}$ & \% & \multirow{2}{*}{$\begin{array}{c}\text { Grande } \\
\text { Área }\end{array}$} \\
\hline Ciência da Computação & Ciências Exatas e da Terra & 8 & 50,0 & \multirow{2}{*}{62,6} \\
\hline Matemática & Ciências Exatas e da Terra & 2 & 12,6 & 6 \\
\hline Linguística & Linguística, Letras e Artes & 3 & 18,8 & 18,8 \\
\hline Psiquiatria e Psicologia & Ciências da Saúde & 1 & 6,2 & 6,2 \\
\hline Biologia & Ciências Biológicas & 1 & 6,2 & 6,2 \\
\hline Pedagogia & Ciências Humanas & 1 & 6,2 & 6,2 \\
\hline Total & & $\mathbf{1 6}$ & \multicolumn{1}{|c|}{$\mathbf{1 0 0 , 0}$} \\
\hline
\end{tabular}

Fonte: os autores.

\subsection{QP5: Quais são os objetivos do uso dos chatbots na educação?}

A resposta para essa questão de pesquisa deu-se por meio da análise dos termos utilizados pelos autores para descrever os objetivos. Os resultados demonstram uma distribuição aproximadamente uniforme dos objetivos dos estudos, com maior frequência em: implementar um chatbot para uso educacional (5); utilizar um chatbot como tutor inteligente (5); propor uma metodologia própria de desenvolvimento(4); criar um chatbot adaptativo (4); realizar a avaliação do chatbot (3); autoaprendizagem (3); aprendizagem colaborativa (3) e mediação da aprendizagem (3). Para fins desta análise, os artigos não foram categorizados de forma exclusiva.

\subsection{QP6: Quais são as principais técnicas e tecnologias que vêm sendo utilizadas na implementação de chatbots na educação?}

Atualmente existem várias ferramentas e plataformas de desenvolvimento de chatbots (Nivamat; Champaneria, 2017). Embora todas sigam uma arquitetura básica, diferentes técnicas e tecnologias mostram-se adequadas para tipos específicos de chatbots. Para fins desta pesquisa, com relação às técnicas, entende-se as linguagens de programação utilizadas, tanto na criação da base de conhecimento quanto no desenvolvimento do sistema; já com relação à tecnologia, considera-se as plataformas e ferramentas utilizadas no desenvolvimento do sistema.

No que se refere às técnicas utilizadas, sete $(43,8 \%)$ trabalhos informaram que utilizaram AIML (Artificial Intelligence Markup Language). Cinco (31,2\%) usaram abordagens baseadas em linguagem natural, como pattern matching, tabelas Hash para keywords, regras de produção em padrões de perguntas-respostas, ferramentas de reconhecimento de fala e sua própria linguagem de marcação alternativa ao AIML com gramáticas. Um artigo $(6,2 \%)$ modelou um sistema especialista usando regras de produção e raciocínio baseado em casos. Um $(6,2 \%)$ usou um framework de agentes colaborativos com suporte a conversação. O restante $(12,6 \%)$ não especificou a técnica.

Com relação às linguagens de programação utilizadas no desenvolvimento do sistema como um todo, apenas 4 artigos $(25,0 \%)$ informaram, dividindo-se em Java (2 $12,5 \%)$, C\# $(1-6,2 \%)$ e PHP $(1-6,2 \%)$.

Quanto às tecnologias empregadas, observou-se nas publicações extraídas que grande parte das soluções usa plataformas Web. Dentre os 16 trabalhos analisados, 2 $(12,5 \%)$ utilizaram o Infochat; 3 (18,8\%) usaram o Program-O; o Program-D foi usado em 2 (12,5\%), um $(1-6,2 \%)$ utilizou PandoraBots; um $(1-6,2 \%)$ utilizou o Bazzar, enquanto o restante $(7-43,8 \%)$ não identificou a plataforma utilizada ou desenvolveram sua própria solução. 
Em uma análise adicional, observou-se que as interfaces utilizadas são em sua maioria Web, com apenas duas publicações que abordaram o uso de dispositivos móveis, uma utilizando o chatbot pelo WhatsApp (ID 9) - (Moreno et al., 2015) e outra onde o ambiente se adapta a diferentes tipos de dispositivos computacionais (ID 15) - (Paschoal, Chicon e Falkembach, 2016).

\subsection{QP7: Quais foram os principais resultados observados nos trabalhos analisados?}

Mediante a análise detalhada dos 16 artigos extraídos, levantou-se os principais resultados obtidos pelos estudos, relacionados sucintamente junto a seus objetivos no Quadro 5. Apesar da diversidade de áreas de conhecimento e público-alvo, observa-se, em geral, conclusões positivas quanto à aplicação dos chatbots para fins educacionais, bem como a identificação dos pontos que devem ser considerados para maior fluidez na interação com os usuários.

Quadro 5 - Principais resultados encontrados de acordo com os objetivos dos artigos analisados

\begin{tabular}{|c|c|c|c|}
\hline ID & Autores & Objetivo & Principais resultados \\
\hline 1 & $\begin{array}{l}\text { Okita } \\
(2014)\end{array}$ & $\begin{array}{l}\text { Comparação entre } \\
\text { autoaprendizado com e } \\
\text { sem chatbot. }\end{array}$ & $\begin{array}{l}\text { Os alunos que utilizaram o chatbot obtiveram } \\
\text { melhores resultados na aprendizagem. }\end{array}$ \\
\hline 2 & $\begin{array}{l}\text { Latham et } \\
\text { al. } \\
(2012)\end{array}$ & $\begin{array}{l}\text { Proposta de tutor } \\
\text { inteligente que se adapta } \\
\text { ao estilo de aprendizagem } \\
\text { do estudante. }\end{array}$ & $\begin{array}{l}\text { Capacidade de predição do estilo de } \\
\text { aprendizagem com acurácia superior a } 61 \% \text {. }\end{array}$ \\
\hline 3 & $\begin{array}{l}\text { Jia et al. } \\
(2012)\end{array}$ & $\begin{array}{l}\text { Avaliação individualizada } \\
\text { do aprendizado de inglês } \\
\text { como segunda língua. }\end{array}$ & $\begin{array}{l}\text { Melhoria significativa nas habilidades de } \\
\text { compreensão, pronúncia, escrita e vocabulário. }\end{array}$ \\
\hline 4 & $\begin{array}{l}\text { Tegos, } \\
\text { Demetriadis } \\
\text { e Karakostas } \\
(2015)\end{array}$ & $\begin{array}{l}\text { Impacto do chatbot em } \\
\text { atividades colaborativas } \\
\text { entre alunos. }\end{array}$ & $\begin{array}{l}\text { Maior engajamento dos alunos em diálogos mais } \\
\text { produtivos; argumentação explícita nas atividades } \\
\text { colaborativas; melhoria na aprendizagem com a } \\
\text { intervenção do agente na argumentação entre os } \\
\text { alunos. }\end{array}$ \\
\hline 5 & $\begin{array}{l}\text { Latham, } \\
\text { Crockett e } \\
\text { McLean } \\
(2014)\end{array}$ & $\begin{array}{l}\text { Proposta de sistema para } \\
\text { adaptação ao estilo de } \\
\text { aprendizagem do } \\
\text { estudante. }\end{array}$ & $\begin{array}{l}\text { Melhores resultados no desempenho dos alunos } \\
\text { que utilizaram o sistema de tutoria com chatbot } \\
\text { conforme seu estilo de aprendizagem. }\end{array}$ \\
\hline 6 & $\begin{array}{l}\text { Schouten et } \\
\text { al. } \\
(2018)\end{array}$ & $\begin{array}{l}\text { Aplicação de técnicas } \\
\text { afetivas em um tutor } \\
\text { digital para alunos com } \\
\text { baixa alfabetização. }\end{array}$ & $\begin{array}{l}\text { Verificação da importância da personalização do } \\
\text { chatbot para a aprendizagem. }\end{array}$ \\
\hline 7 & $\begin{array}{l}\text { Dyke et al. } \\
\text { (2013) }\end{array}$ & $\begin{array}{l}\text { Uso de chatbots na } \\
\text { mediação do aprendizado } \\
\text { colaborativo. }\end{array}$ & $\begin{array}{l}\text { Resultados significativos no aprendizado entre } \\
\text { alunos que foram mediados pelo chatbot. }\end{array}$ \\
\hline 8 & $\begin{array}{l}\text { Aguiar, Taro } \\
\text { uco } \\
\text { e Reategui } \\
(2011)\end{array}$ & $\begin{array}{l}\text { Apoio aos estudantes em } \\
\text { um sistema de } \\
\text { aprendizagem } \\
\text { autorregulada. }\end{array}$ & $\begin{array}{l}\text { Aprimoramento de habilidades cognitivas e } \\
\text { construção do conhecimento, porém sem aumento } \\
\text { do engajamento. }\end{array}$ \\
\hline 9 & $\begin{array}{l}\text { Moreno et } \\
\text { al. } \\
(2015)\end{array}$ & $\begin{array}{l}\text { Proposta de chatbot para } \\
\text { uso na área de linguística- } \\
\text { Atlas Brasileiro. }\end{array}$ & $\begin{array}{l}\text { Vantagem da utilização de chatbot através de } \\
\text { aplicativos populares de mensagem instantânea. } \\
\text { Aceitação positiva por parte dos alunos. }\end{array}$ \\
\hline 10 & $\begin{array}{l}\text { Oliveira } e t \\
\text { al. } \\
(2010)\end{array}$ & $\begin{array}{l}\text { Proposta de chatbot com } \\
\text { intenção e personalidade, } \\
\text { usando ontologias, para } \\
\text { apoio ao ensino e } \\
\text { aprendizagem em } \\
\text { psiquiatria e psicologia. }\end{array}$ & $\begin{array}{l}\text { Aceitação positiva pelos alunos no apoio ao } \\
\text { ensino e aprendizagem. Arquitetura escalável do } \\
\text { agente possibilita conversação com maior } \\
\text { coerência através do uso de ontologias. }\end{array}$ \\
\hline 11 & $\begin{array}{l}\text { Lemos, } \\
\text { Campos e } \\
\text { Nunes }\end{array}$ & $\begin{array}{l}\text { Apresentar a análise } \\
\text { comportamental de um }\end{array}$ & $\begin{array}{l}\text { Identificação de fatores que aprimoram a } \\
\text { consistência do diálogo do chatbot: linguagem } \\
\text { informal, expressar emoções, identificar }\end{array}$ \\
\hline
\end{tabular}




\begin{tabular}{|c|l|l|l|}
\hline & $(2012)$ & $\begin{array}{l}\text { chatbot desenvolvido para } \\
\text { ensinar algoritmos. }\end{array}$ & $\begin{array}{l}\text { redundâncias de linguagem e possuir aspectos } \\
\text { humanos para ser considerado consistente. }\end{array}$ \\
\hline $\mathbf{1 2}$ & $\begin{array}{l}\text { Shopf e } \\
\text { Duarte } \\
(2005)\end{array}$ & $\begin{array}{l}\text { Proposta de um chatbot } \\
\text { que emprega linguagem } \\
\text { regional. }\end{array}$ & $\begin{array}{l}\text { Demonstrou ganho cognitivo do aluno de forma } \\
\text { significativa, motivação por parte do aluno pelo } \\
\text { uso de linguagem bem humorada do chatbot. }\end{array}$ \\
\hline $\mathbf{1 3}$ & $\begin{array}{l}\text { Paschoal, } \\
\text { Chicon e } \\
\text { Falkembach } \\
(2016)\end{array}$ & $\begin{array}{l}\text { Proposta de } \text { chatbot } \\
\text { integrado ao AVA } \\
\text { Moodle, integrando } \\
\text { conceitos de aprendizagem } \\
\text { ubíqua. }\end{array}$ & $\begin{array}{l}\text { Adaptação do chatbot ao nível de conhecimento e } \\
\text { desempenho do estudante, e diferentes tipos de } \\
\text { dispositivos computacionais. }\end{array}$ \\
\hline $\mathbf{1 4}$ & $\begin{array}{l}\text { Leonhardt } \text { et } \\
\text { al. } \\
(2003)\end{array}$ & $\begin{array}{l}\text { Proposta de } \text { chatbot para } \\
\text { ensino nas áreas de física e } \\
\text { redes de computadores. }\end{array}$ & $\begin{array}{l}\text { Participação mais ativa dos estudantes e foco do } \\
\text { diálogo no interlocutor. Observação da } \\
\text { necessidade de muitas interações pelos alunos, e } \\
\text { consequente análise dos registros desta utilização } \\
\text { para a criação de novas categorias, tornando a } \\
\text { conversação mais natural e abrangente. }\end{array}$ \\
\hline $\mathbf{1 5}$ & $\begin{array}{l}\text { Paschoal, } \\
\text { Chicon e } \\
\text { Falkembach } \\
\text { (2017) }\end{array}$ & $\begin{array}{l}\text { Proposta de } \text { chatbot } \\
\text { integrado a ambiente } \\
\text { virtual de aprendizagem. }\end{array}$ & $\begin{array}{l}\text { Contribuição para a aprendizagem dos alunos. } \\
\text { Identificação de fatores por parte dos alunos que } \\
\text { deveriam ser aprimorados, como ampliação da } \\
\text { base de conhecimento e tratamento de algumas } \\
\text { sentenças de interação. }\end{array}$ \\
\hline $\mathbf{1 6}$ & $\begin{array}{l}\text { Gomes, } \\
\text { Barbosa e } \\
\text { Geyer } \\
\text { (2005) }\end{array}$ & $\begin{array}{l}\text { Proposta de agente } \\
\text { pedagógico integrado a } \\
\text { ambiente virtual de } \\
\text { aprendizagem. }\end{array}$ & $\begin{array}{l}\text { Contribuição do chatbot para tornar o ambiente } \\
\text { virtual mais interativo e participativo. }\end{array}$ \\
\hline
\end{tabular}

Fonte: os autores.

Observa-se que os resultados dos artigos analisados circularam em torno da melhoria da aprendizagem, das habilidades e do engajamento dos estudantes, o que incentiva e embasa o uso de chatbots na educação. Também foram apontadas melhorias necessárias para a aplicação das ferramentas, como personalização e aumento na base de conhecimento de chatbots.

\section{Conclusões}

Este trabalho apresentou uma Revisão Sistemática de Literatura sobre o uso de chatbots com objetivos educacionais, cobrindo o período entre os anos de 2000 a 2017 em 12 bases de dados diferentes, nacionais e internacionais. Na busca inicial 159 publicações foram encontradas, restando apenas 16 após a aplicação dos critérios de exclusão e inclusão.

Entre outros resultados, foi possível constatar que apesar de a maior parcela de estudos serem voltados para o uso dos chatbots em áreas do ensino específicas, principalmente na área da Ciência da Computação, também existem pesquisas que buscam construir um sistema flexível, com uma lógica genérica, que pode servir de modelo ou se encaixar em diversas disciplinas, ou que tenha um propósito educacional, como estimular a metacognição ou autorregulação (ID 8) - (Aguiar, Tarouco e Reategui, 2011), e incentivar o aprendizado colaborativo (ID 7) - (Dyke et al., 2013). Isto pode ser uma vantagem no âmbito educacional, permitindo que uma mesma ferramenta beneficie diversas áreas de aplicação.

De forma semelhante, o nível educacional mais presente nas pesquisas foi o do ensino superior, possivelmente justificado pela maior adoção de ferramentas tecnológicas neste nível educacional, mas foram encontrados estudos que implementaram chatbots que podem ser adaptáveis para outros níveis de ensino (ID 6) - (Schouten et al., 2018). Assim, a ferramenta acaba tendo maior alcance podendo ser utilizada por um público maior; porém, requer considerável esforço adicional, já que a comunicação do chatbot deve ser adequada aos níveis de ensino e áreas envolvidas, o que envolve diferentes faixas etárias 
e características socioculturais. Entretanto, não se observou a aplicação do mesmo chatbot em mais de um nível educacional simultaneamente.

Foi possível perceber que o Brasil tem uma participação significativa no conhecimento acadêmico no que tange aos objetivos desta RSL, pois das seis bases de dados de origem dos artigos extraídos, duas são nacionais. Neste contexto, 37,5\% dos artigos analisados encontravam-se em bases nacionais e $62,5 \%$ em bases internacionais. Com relação ao idioma, $50 \%$ das publicações foram escritas em inglês e $50 \%$ em português. Porém, os artigos em inglês são mais extensos, e corresponderam a $72 \%$ do corpus analisado.

Os resultados obtidos com a RSL denotam a oportunidade de pesquisa e inovação, pois o número de estudos extraídos foi bem abaixo da expectativa dos pesquisadores. Pesquisas que culminam em aplicações educacionais são mais complexas e recebem poucos subsídios, enquanto que grande parcela dos chatbots disponíveis na internet são para fins comerciais, pelo volume de investimentos e do retorno financeiro. Contudo, os trabalhos existentes são de qualidade, ajudam e incentivam a expansão do conhecimento na área. De acordo com a análise dos artigos, entre os principais fatores que impedem a difusão do uso de chatbots na educação estão: a necessidade de uma base de conhecimento considerável para uma conversa satisfatória; a maior complexidade e imprevisibilidade do fluxo de diálogo entre o agente e o aluno em relação a uma conversação casual.

A linguagem de marcação AIML foi a técnica mais utilizada no desenvolvimento de chatbots educacionais, por ser de fácil aprendizagem e implementação. Atualmente, técnicas tradicionais de IA se renovam, pela evolução tecnológica, abordando técnicas de aprendizado de máquina e processamento de linguagem natural, tais como Word2Vec (Mikolov et al., 2013) e Redes Neurais Recorrentes (Hopfield, 1982), recentemente empregadas no desenvolvimento do chatbot proprietário da IBM - Watson (Gliozzo et al., 2017) e TensorFlow (Abadi et al., 2016), para citar apenas alguns, que apresentam soluções eficientes; e que, por sua vez, não foram utilizadas em nenhuma das soluções apresentadas nas publicações extraídas, possivelmente porque requerem um maior investimento financeiro.

Como perspectivas futuras, esta RSL compõe um substancial aporte teórico para um projeto mais amplo e profundo na área. A aplicação de um chatbot como tutor para o ensino de Trigonometria, aliando tecnologias de Deep Learning, processamento de linguagem natural, sistemas tutores inteligentes e agentes conversacionais baseados no Watson (Gliozzo et al., 2017), tendo como público-alvo alunos de cursos superiores de engenharia.

\section{Referências}

ABADI, M. et al. TensorFlow: A system for large-scale machine learning. In: Proceedings of the 12th USENIX Symposium on Operating Systems Design and Implementation, p. 265283, 2016.

AGUIAR, Eliane Vigneron Barreto; TAROUCO, Liane Margarida Rockenbach; REATEGUI, Eliseo Berni. A Construção do conhecimento matemático com engajamento e aprimoramento de habilidades cognitivas apoiada por um agente conversacional. RELATEC-Revista Latinoamericana de Tecnologia Educativa.Vol 10,p.21-35, 2011.

BERNARDINI, Andréia A.; SÔNEGO, Arildo A.; POZZEBON. Eliane. CHATBOTS: Uma análise bibliométrica do estado da arte da literatura. ARTEFactum - Revista de Estudos de Linguagens e tecnologias. Ano X, n.1. 2018. Disponível em: < http://artefactum.rafrom. com.br/index.php/artefactum/article/view/1579/777 >. Acesso em: 10 abr. 2018. 
BRADEŠKO, Luka; MLADENIĆ, Dunja. A survey of chatbot systems through a Loebner Prize Competition. In: Proceedings of Slovenian Language Technologies Society Eighth Conference of Language Technologies, p. 34-37, 2012.

DALE, Robert. The commercial NLP landscape in 2017. Natural Language Engineering, v. 23, n. 4, p. 641-647, 2017.

DE GASPERIS, G.; CHIARI, I.; FLORIO, N. AIML knowledge base construction from text corpora. In Artificial intelligence, evolutionary computing and metaheuristics (pp. 287-318). Springer, Berlin, Heidelberg, 2013.

DYKE, Gregory; ADAMSON, David; HOWLEY, Iris; ROSÉ, Carolyn Penstein . Enhancing Scientific Reasoning and Discussion with Conversational Agents. IEEE Transactions on Learning Technologies, Vol. 6, No. 3, 2013.

GLIOZZO, A. et al. Building Cognitive Applications with IBM Watson Services. IBM Redbooks. 2017. 132 p.

HOPFIELD, J. J. Neural networks and physical systems with emergent collective computational abilities. In: Proceedings of the National Academy of Sciences, v. 79, n. 8, p. 2554-2558, 1982. KITCHENHAM, B.; CHARTERS, S.; BUDGEN, D.; BRERETON, P.; TURNER, M.; LINKMAN, S.; JORGENSEN, M.; MENDES, E.; VISAGGIO, G. Guidelines for performing Systematic Literature Reviews in Software Engineering. 2007. EBSE Technical Report, Keele University and University of Durham.

LEONHARDT, M. D.; CASTRO, D. D.; DUTRA, R. L. S.; TAROUCO, L. M. R. Elektra: um chatterbot para uso em ambiente educacional. RENOTE, v. 1, n. 2, 2003. Disponível em: < http://seer.ufrgs.br/index.php/renote/article/view/14336/8251 >Acesso em : 10 out. 2017.

MASCHE, J., \& Le, N. T. A Review of Technologies for Conversational Systems. In International Conference on Computer Science, Applied Mathematics and Applications (pp. 212-225). Springer, Cham, 2017.

MIKOLOV, T.; CHEN, K.; CORRADO, G.; DEAN, J. Efficient estimation of word representations in vector space. ICLR Workshop, 2013.

MORAES, Sílvia M. W.; DE SOUZA, Luciano Severo. Uma Abordagem Semiautomática para Expansão e Enriquecimento Linguístico de Bases AIML para Chatbots. In: Congresso Internacional de Informática Educativa, 20., 2015, Santiago. Anais. Santiago: Universidad de Chile, p. 600-605, 2015.

MORENO,Fábio; MANFIO, Edio ; BARBOSA, Cinthyan Renata; BRANCHER, Jacques Duílio. Tical: Chatbot sobre o Atlas Linguístico do Brasil no WhatsApp. In: Brazilian Symposium on Computers in Education (Simpósio Brasileiro de Informática na Educação - SBIE), p. 279, 2015.

NIVAMAT, K.; CHAMPANERIA, T. Chatbots: An overview Types, Architecture, Tools and Future Possibilities. In: IJSRD - International Journal for Scientific Research \& Development. v.5, n. 7, 2017.

SEWALD JUNIOR, Egon; ROVER, Aires J.; MACHADO, Nivaldo; BRAIDA, Celso R.; DA SILVA, Edson R. G. Chatterbots em língua portuguesa: Problema da quebra de sentido e as categorias ontológicas. Anais do Computer on the Beach, p. 249-258, 2011.

SGOBBI, Fabiana S.; NUNES, Felipe B.; BOS, Andreia S.; BERNARDI, Giliane; TAROUCO, Liane M. R. Interação com artefatos e personagens artificiais em mundos virtuais. In: Brazilian Symposium on Computers in Education (Simpósio Brasileiro de Informática na Educação - SBIE), p. 642, 2014.

SCHOUTEN, Dylan G.M. , VENNEKER,Fleur; BOSSE, Tibor; NEERINCX, Mark A.; CREMERS , Anita H.M. A Digital Coach That Provides Affective And Social Learning Support To Low-Literate Learners. IEEE Transactions on Learning Technologies, v. 11, 2018.

SOLIMAN, M.; GUETL, C.. Intelligent pedagogical agents in immersive virtual learning environments: A review. In: MIPRO, 2010 proceedings of the 33rd international convention. IEEE, p. 827-832, 2010.

WEIZENBAUM, Joseph. ELIZA - a computer program for the study of natural language communication between man and machine. Communications of the ACM, v. 9, n. 1, p. 36-45, 1966. 